\section{Cahiers de Narratologie}

Analyse et théorie narratives

8 | 1997

Création de l'espace et narration littéraire

\title{
La dimension spatiale du récit dans le Quichotte
}

Jean-Louis Brau

\section{CpenEdition}

Journals

Édition électronique

URL : http://journals.openedition.org/narratologie/10727

DOI : 10.4000/narratologie. 10727

ISSN : 1765-307X

Éditeur

LIRCES

Édition imprimée

Date de publication : 1 décembre 1997

Pagination : 81-94

ISBN : 291089746X

ISSN : 0993-8516

Référence électronique

Jean-Louis Brau, «La dimension spatiale du récit dans le Quichotte», Cahiers de Narratologie [En ligne], 8 | 1997, mis en ligne le 01 décembre 2020, consulté le 25 février 2021. URL : http://

journals.openedition.org/narratologie/10727 ; DOI : https://doi.org/10.4000/narratologie.10727

Ce document a été généré automatiquement le 25 février 2021.

Article L.111-1 du Code de la propriété intellectuelle. 


\title{
La dimension spatiale du récit dans le Quichotte
}

\author{
Jean-Louis Brau
}

1 En écrivant le Quichotte, l'intention première de Cervantès, clairement affirmée dès le prologue et abondamment confirmée tout au long de l'histoire de l'ingénieux hidalgo, a été celle de parodier les livres de chevalerie, si appréciés du public de l'époque et si critiqués par les censeurs. Répéter cette évidence ne présenterait aucun intérêt si elle ne constituait la base indispensable d'une étude des structures narratives de l'œuvre, et, en l'occurrence, de la dimension spatiale du récit.

2 Pour parodier, il faut commencer par imiter ${ }^{1}$, et $c^{\prime}$ est ce que fait Cervantès quand il utilise la structure narrative et la thématique des livres de chevalerie, mais en les transposant dans un environnement espagnol et dans une tonalité burlesque. On trouvera donc logiquement dans le Quichotte diverses caractéristiques spatiales du récit chevaleresque. Les deux types de récit sont construits autour du motif du voyage, le plus universellement utilisé dans tous les romans d'aventures et qui prend ici la forme du vagabondage du chevalier. Dans tous les livres de chevalerie, les lieux de la diégèse sont répartis inégalement entre les espaces ouverts et les espaces fermés. Les premiers sont de loin les plus nombreux puisque les héros se trouvent la plupart du temps en rase campagne. Les espaces fermés sont, presque toujours, des châteaux ou des palais, mais les héros n'y séjournent pas longtemps car ils passent le plus clair de leur temps à chercher des aventures glorieuses dans des lieux déserts.

Dans ces histoires fabuleuses, la description des lieux, qu'il s'agisse de lieux ouverts ou fermés, n'existe pratiquement pas. Le narrateur se contente de fournir des indications très générales pour définir le cadre. L'action se situe dans une plaine, une vallée, une montagne, une forêt, un chemin ou un château et, chaque fois, le terme le plus vague et le plus générique suffit. Il n'y a aucun détail qui donne une précision quelconque.

4 Cette extrême imprécision traduit diverses préoccupations de la part de l'auteur. D'abord celle de privilégier l'action et les sentiments au détriment de la description qui retarderait un récit épique toujours animé, riche en péripéties violentes ou sentimentales. Ensuite, c'est le topos de l'exotisme qui empêche l'auteur de situer son 
histoire dans des lieux connus et donc de donner le plus petit détail qui puisse rattacher le récit à une quelconque réalité géographique ou historique qui soit familière au lecteur. Au contraire, la plupart des histoires de chevalerie ont pour cadre un pays très lointain dans l'espace et/ou dans le temps: l'empire de Trébizonde, la Grande Bretagne "Quelques années après la passion de notre rédempteur et sauveur Jésus Christ $»^{2}$, Constantinople, le Caucase, l'Inde, etc. Cet exotisme combiné à la parcimonie des références spatiales laisse le champ libre à l'imagination du lecteur qui peut ainsi s'évader de sa réalité quotidienne insipide.

5 La pastorale reprend la même formule et la simplifie en réduisant encore plus les éléments du cadre. Ici, une prairie, un bois, un ruisseau ou une source suffisent à composer le paysage dans lequel bergers et bergères vivent leurs amours contrariées. On reconnaît là le vieux locus amoenus qui constitue, selon les termes de Curtius : "depuis l'époque de l'Empire romain jusqu'au XVIe siècle, le motif central de toutes les descriptions de la nature ${ }^{3}$. Ce topos spatial se répète indéfiniment dans le roman pastoral dont il constitue le cadre quasi exclusif, et on le rencontre même dans la littérature épique, par exemple dans le Poema de mio Cid ou dans les livres de chevalerie ${ }^{4}$. Il exprime une idée de bien-être, de plaisir et de repos avec une telle économie de moyens qu'il correspond à une image presque abstraite, fortement stéréotypée, du paysage idéal. Cela revient à dire que la fonction mimétique de la description, c'est-à-dire la production de l'illusion réaliste, ou autrement dit la " narraticité ${ }^{5}$ des lieux de l'histoire, est quasi inexistante, ce qui se comprend dans une littérature idéaliste à l'extrême et qui veut avant tout que le lecteur puisse s'évader de son espace et/ou de son époque ${ }^{6}$.

6 Cervantès coule son récit dans le moule formel des livres de chevalerie et du roman pastoral. C'est ainsi qu'il commence par utiliser leur cadre spatial qu'il transpose dans une topographie espagnole indiquée par quelques toponymes: la Manche, la Sierra Morena et l'Andalousie pour le Quichotte de 1605, de nouveau la Manche puis la Catalogne pour le Quichotte de 1615. A ces noms de régions s'ajoutent quelques noms de localités, très rares dans la narration principale (c'est-à-dire l'histoire de don Quichotte): Puerto Lápice, El Toboso, Barcelone, un peu plus nombreux dans les nouvelles intercalées: Séville, Florence, Alger, Constantinople, Tunis, La Goulette, Vélez Malaga et Madrid ${ }^{7}$. Mais cet ancrage spatial et très faible et très vague. C'est ce qui se passe au tout début du roman dans la célébrissime phrase :

Dans un endroit de la Manche, dont je ne veux point me rappeler le nom ${ }^{8}$.

7 Avec cette phrase qui a fait couler tant d'encre Cervantès donne le ton. L'ancrage spatial lui importe vraiment peu et la plupart du temps il s'en désintéresse totalement, en particulier dans les nouvelles intercalées dont l'intrigue est souvent située dans un lieu indéterminé, dans un village proche ou dans une ville dont les noms ne sont même pas mentionnés.

8 En ce qui concerne les espaces fermés, relativement rares, on ne sait pas ou sont situés la fameuse auberge de Juan Palomeque le Gaucher ou le palais des Ducs qui jouent un rôle si important dans l'histoire, ou encore la maison du Chevalier au Vert Manteau. Ce n'est qu'à la fin du roman que la maison de don Antonio Moreno est située à Barcelone, mais sans plus de précision. Dans ce roman si long, la topographie mimétique est donc réduite à sa plus simple expression, contribuant ainsi très peu à l'illusion référentielle, indispensable pour susciter l'adhésion du lecteur. Cette grande indétermination topographique est-elle au moins compensée par une plus grande précision dans la 
description des lieux ? Pour le savoir, il faut analyser la toposémie fonctionnelle du récit, c'est-à-dire faire une étude sémiologique des lieux de l'histoire9

On peut d'abord observer que le phénomène d'intertextualité entre le Quichotte et les grands genres littéraires mentionnés plus haut, les livres de chevalerie et le roman pastoral, fonctionne ici parfaitement, comme on pouvait s'y attendre. En effet, les lieux ouverts et déserts forment le cadre privilégié des aventures de don Quichotte. Si on les compare avec les lieux fermés (maisons, auberges, agglomérations), un calcul approximatif fait ressortir l'indéniable majorité des premiers, deux fois plus nombreux, c'est-à-dire à peu près soixante contre trente.

Comment ces lieux sont-ils décrits ? Également de la manière la plus vague. On ne peut pas parler ici de descriptions parce qu'elles sont presque totalement absentes du récit. Pour les lieux ouverts, Cervantès utilise un lexique très limité composé des termes génériques suivants : un chemin, la campagne, un bois, une prairie, une montagne, un fleuve, une source, des arbres, des rochers, la mer, la plage. Les rares précisions qu'il consent à donner concernent les arbres qui sont la plupart du temps des chênes verts ou des chênes-lièges et, dans le cas des nouvelles intercalées de tonalité pastorale, c'est-à-dire deux fois, des hêtres. Les seules descriptions que l'on peut trouver dans tout le Quichotte sont des pseudo-descriptions, soit à peine ébauchées et immédiatement éludées, soit totalement topiques.

La présentation de la maison du Chevalier au Vert Manteau, don Diego de Miranda, au chapitre dix-huit de la deuxième appartient au premier type. Après avoir donné quelques détails sur l'entrée de la maison, le narrateur élude la description proprement dite en justifiant cette ellipse par une déclaration assez paradoxale qui explique l'absence de descriptions dans le roman par le souci de "vérité » qui anime l'auteur et qui semble contraire à tout « effet de réalité » tel que nous le concevons aujourd'hui :

L'auteur peint ici tous les aspects de la maison de don Diego en nous décrivant ainsi ce que contient la maison d'un gentilhomme campagnard et riche; mais le traducteur de cette histoire jugea bon de passer sous silence ces détails et d'autres semblables, parce qu'ils ne correspondaient pas au propos principal de l'histoire; laquelle tire sa force de la vérité plutôt que des froides digressions ${ }^{10}$.

Cette phrase significative résume bien la conception cervantine du roman qui privilégie l'action et la psychologie des personnages au détriment du réalisme descriptif. Mais, pour en revenir à l'espace, le second type de description peut se subdiviser en deux topoï, le locus amoenus et le château idéal, inégalement répartis dans le récit. On y trouve beaucoup plus souvent le locus amoenus tandis que le château idéal n'apparaît qu'une ou deux fois.

Les mêmes caractéristiques du locus amoenus définies plus haut à propos des livres de chevalerie et du roman pastoral se retrouvent aussi dans les nombreuses occurrences de ce vieux topos dans le Quichotte. Trois éléments de base, inlassablement répétés, constituent ce paysage fortement stéréotypé dont la «narraticité » est proche de zéro. Ce sont le pré ou la prairie avec son herbe verte et tendre et parfois des fleurs, le ruisseau aux eaux cristallines et des arbres qui donnent une ombre propice au repos à l'heure de la sieste dans la fournaise de l'été de la Manche ou de l'Andalousie. Les trois éléments peuvent coexister :

Ils arrivèrent, tout en devisant, au pied d'une haute montagne... Un ruisseau paisible coulait sur sa pente, et tout à l'entour il y avait un pré si vert et si luxuriant, qu'il réjouissait les yeux de ceux qui le contemplaient. Il y avait là 
beaucoup d'arbres sauvages et des plantes et des fleurs qui rendaient le lieu agréable ${ }^{11}$. cinquante de cette première partie lorsque don Quichotte imagine pour le chanoine de Tolède l'aventure chevaleresque du lac de poix bouillante dans lequel un chevalier découvre un éblouissant château d'or et pierres précieuses où il est accueilli comme un prince par de belles damoiselles. La description est assez longue, mais don Quichotte l'abrège en signalant, ici aussi, son caractère topique et stéréotypé :

... si je me mettais maintenant à en parler (des costumes des damoiselles) comme les histoires nous les décrivent, on n'en finirait jamais... ${ }^{15}$ c'est-à-dire de constituer un cadre pour l'action et, dans ce roman itinérant celle de permettre de multiples rencontres qui suscitent autant d'aventures. Je n'insiste pas, ceci est par trop évident. Mais en même temps, ces lieux ouverts sont des indices qui ont une fonction narrative et qui, par leurs caractéristiques, même si elles sont indiquées de façon sommaire, programment dans ses grandes lignes l'épisode auquel elles servent de cadre, fonctionnant alors comme des embrayeurs narratif ${ }^{17}$. C'est ainsi que les chemins de plaine, en rase campagne, favorisent les hallucinations de don Quichotte parce qu'il aperçoit au loin des gens ou des objets qu'il transforme, dans sa folie, mais aussi à cause de la distance combinée à la mauvaise visibilité due à la poussière, à la chaleur ou à l'obscurité, en motifs d'aventures chevaleresques. C'est le cas d'un grand nombre d'épisodes comme ceux des moulins à vent, des troupeaux, du barbier, du cortège funèbre, etc... ${ }^{18}$ De leur côté, les montagnes escarpées ou les forêts sont, comme dans les livres de chevalerie ou dans le roman pastoral, des lieux propices aux rencontres mystérieuses qui éveillent la curiosité, comme celle du moulin à foulons, de Cardenio, de Dorotea, du chevrier Eugenio, du chevalier aux miroirs, etc... ${ }^{19}$ 
Prenons un exemple entre mille, celui de la première nouvelle intercalée dans la première partie. C'est le récit de l'enterrement du poète-berger Chrysostome qui a lieu loin du village «dans la campagne, comme s'il était maure, au pied du rocher où se trouve la source du chêne-liège. $»^{20} \mathrm{Ce}$ lieu, environné de hautes montagnes, est un lieu étrange pour un enterrement, comme le souligne le texte. Il prépare, par sa disposition, un rocher qui surplombe la tombe, l'apparition dramatique de Marcelle qui va adresser un discours véhément aux prétendants qu'elle domine, matériellement et moralement, du haut de cette tribune sauvage.

Quant au locus amoenus, avec ses caractéristiques sommaires et stéréotypées, il programme lui aussi le récit car, s'il constitue un informant sur le cadre bucolique dans lequel va se dérouler l'action, c'est surtout un indice qui annonce au lecteur un épisode de tonalité pastorale. Ce cadre bucolique, dans lequel vivent les chevriers qui accueillent don Quichotte et Sancho la veille de l'enterrement d'un berger, annonce l'histoire sentimentale de Marcelle et Chrysostome. L'autre histoire pastorale intercalée, celle de Léandre, est introduite, de la même façon, par le récit d'une halte de don Quichotte et de tous ceux qui l'accompagnent dans une prairie à l'ombre de quelques arbres, lieu propice pour le repos et pour un repas champêtre, mais qui annonce infailliblement un épisode pastoral. En effet, arrive un chevrier qui court derrière une chèvre en l'injuriant, ce qui intrigue les voyageurs ; interrogé par ceux-ci, le chevrier conte alors l'histoire de Léandre.

La programmation du récit par la description sommaire et stéréotypée du locus amoenus et du château est possible et fonctionne ici parfaitement grâce aux connaissances « encyclopédiques» du lecteur (ou de l'auditeur) habitué à associer automatiquement les lieux en question à un type de contenu narratif et à une tonalité, c'est-à-dire à un genre littéraire bien déterminée ${ }^{21}$. Le narrateur compte donc sur cette compétence du lecteur pour interpréter les données spatiales, rares mais significatives, ce qui lui évite de trop s'attarder dans de longues descriptions oiseuses qui ralentiraient le rythme de l'action et des dialogues et qui, en fin de compte, ne correspondraient pas à l'esthétique de l'époque. Le récit n'en est que plus économique et plus efficace. Mais si le narrateur peut ainsi susciter la collaboration interprétative du lecteur, il peut aussi utiliser la programmation spatiale du récit sur le mode parodique, en changeant «l'horizon d'attente » du lecteur pour l'amuser et s'amuser en même temps à ses dépens. On peut citer, comme exemple de cette technique, le début du chapitre XV de la première partie qui conte, dans un locus amoenus où don Quichotte et Sancho se sont arrêtés pour se reposer, mais dans une tonalité burlesque, l'épisode comique des « amours » déçues de Rossinante avec des juments galiciennes plutôt farouches. Dans ce cas, on pourrait parler de programmation spatiale « déceptive » et parodique.

21 Cependant, si l'aventure attend le chevalier sur tous les chemins et dans toutes les montagnes et les forêts où il erre, elle n'est pas absente des lieux fermés, loin de là. Deux d'entre eux, cadres d'événements extraordinaires, se distinguent par leur importance : l'auberge de Juan Palomeque dans la première partie et le palais des Ducs dans la seconde. Don Quichotte y est la victime d'enchantements qui sont autant de bourles. Pour les protagonistes des nouvelles intercalées dans la première partie, l'auberge perdue dans le désert de la Manche est aussi un lieu enchanté où la Providence les réunit pour transformer leur histoire tragique en tragi-comédie au dénouement euphorique. L'auberge et le palais des Ducs sont donc, chacun à sa manière, deux loca fabulosa, avatars des châteaux des livres de chevalerie ou du palais 
de la sage Félicie de la Diane de MONTEMAYOR dont ils donnent une image inversée, burlesque, mais tout aussi fonctionnelle.

En outre, l'auberge est un lieu mixte, fermé mais isolé, sorte d'oasis au milieu de l'immense lieu ouvert du désert de la Manche et qui réunit dans sa promiscuité des personnages des classes sociales les plus diverses. Les aristocrates de haut lignage comme don Fernando ou don Luis côtoient ici des vilains de bas étage, prostituées ou charretiers. Dans ce lieu paradoxal se produisent des événements tantôt prodigieux, tantôt burlesques. Les premiers permettent les dénouements euphoriques des histoires sentimentales des aristocrates réunis là par un hasard miraculeux. Les seconds déchaînent de périodiques tempêtes de violence et de folie au centre desquelles se retrouve invariablement don Quichotte. L'idéalisme des nouvelles intercalées et le réalisme de la narration principale se mêlent alors et leurs frontières s'effacent parce que si les vilains de l'auberge s'évadent parfois de leur condition misérable en écoutant le récit des aventures chevaleresques qui les font rêver, à l'inverse les aristocrates s'abaissent à participer à une farce grotesque imaginée par le curé et pour laquelle ils se déguisent en fantômes qui ligotent don Quichotte pendant son sommeil et l'enferment dans une cage. Ils le font, certes, dans la louable intention de ramener don Quichotte chez lui pour le guérir de sa folie, mais ceci préfigure, au niveau narratif, les bourles fantastiques inventées par les Ducs et, au niveau symbolique, la profonde dégradation des mœurs de l'aristocratie espagnole de l'époque.

En effet, dans le cas de l'auberge, la parodie des livres de chevalerie réside dans la nature du lieu, misérable et mal fréquenté, qui fonctionne à deux niveaux. Dans la narration principale, le caractère burlesque des aventures que le héros y vit, la plupart du temps à cause de ses hallucinations, constitue la parodie la plus évidente des livres de chevalerie. Mais dans les nouvelles intercalées aussi, le fait même que la Providence ait choisi un lieu aussi vulgaire que l'auberge pour provoquer les miraculeuses rencontres qui permettent les dénouement euphoriques est parodique par rapport aux châteaux des livres de chevalerie dont le narrateur et don donnent les descriptions citées plus haut. Dans le palais des Ducs, haut lieu de la tromperie et de l'illusion, ce sont les Ducs eux-mêmes, aristocrates de haut lignage riches et oisifs, qui imaginent des bourles prodigieuses aux dépens de don Quichotte et de Sancho. Si l'auberge est un lieu providentiel et burlesque dans lequel alternent la violence comique et l'émotion, le palais des Ducs n'est qu'un luxueux théâtre de marionnettes. Le topos spatial de la littérature idéaliste qu'était le prestigieux château des livres de chevalerie est ici un topos dégradé, parodique, qui symbolise la décadence de la haute société. Ainsi, au signifiant spatial correspond, entre autres, un signifié social et idéologique.

Il faudrait ajouter une remarque sur la forme de l'itinéraire des protagonistes, symbolique aussi. Mais, pour me limiter à l'espace réduit de ce travail, je me contenterai d'indiquer que dans le Quichotte l'opposition structurale entre la ligne et le cercle fonctionne de deux manières inverses selon le type d'histoire. Dans la narration principale et dans les nouvelles intercalées de tonalité pastorale, l'itinéraire linéaire est le symbole de la liberté et donc du triomphe du héros ou de l'héroïne qui parviennent à réaliser leurs projets de vie en s'évadant le leur ennuyeux village, alors que le retour au lieu de départ symbolise l'échec du projet personnel. Au contraire, dans les autres nouvelles idéalistes l'itinéraire linéaire symbolise le malheur et l'échec, et l'itinéraire circulaire un dénouement euphorique. Pour les protagonistes, la manière de parcourir $l^{\prime}$ espace correspond donc à leur trajectoire vitale ${ }^{22}$. 
Pour conclure je dirai que malgré la rareté et le caractère répétitif et stéréotypé des éléments qui représentent l'espace dans le Quichotte, leur analyse est instructive. Si la topographie mimétique est très réduite, une étude de la toposémie fonctionnelle montre que les caractéristiques fortement topiques des lieux ouverts, et particulièrement du locus amoenus, programment le récit en l'orientant vers les schémas narratifs des grands genres littéraires dont il s'inspire, les livres de chevalerie et le roman pastoral. Mais, dès l'incipit, la tonalité parodique subvertit la caractérisation sociale et/ou symbolique des lieux en faisant, par exemple, d'une auberge misérable perdue dans le désert de la Manche ou, à l'inverse, du palais d'un Duc, des lieux magiques à l'instar des châteaux des livres de chevalerie ou du palais de la magicienne Félicie, dont ils donnent une image inversée, parodique et burlesque, mais tout aussi fonctionnelle que l'image idéaliste. D'autre part, l'espace fonctionne ici diégétiquement selon l'opposition classique circularité / linéarité, symbolique de l'échec ou du triomphe des personnages mais dans une relation directe ou inversée selon la tonalité parodique ou idéaliste des histoires narrées. En outre, l'ancrage spatial faible constitue l'un des éléments qui confèrent au récit une universalité attestée par le succès jamais démenti de l'œuvre mais sans exclure pour autant un symbolisme idéologique qui perce sous l'apparence neutre des lieux stéréotypés. Je ne peux pas développer ici cette idée et je me contenterai de dire que la parodie de fictions démodées comme les livres de chevalerie ou intemporelles comme le roman pastoral, déjà perceptible dans la dimension spatiale du récit, masque subtilement, sous une tonalité presque toujours burlesque, un discours qui nous parle des problèmes de la société espagnole du Siècle d'Or, de ses castes, de son aristocratie rivole et de la dégradation des idéaux les plus hauts qui avaient fait sa gloire et qui ne s'incarnent plus que dans un vieux fou rossé à chaque instant sur les chemins désertiques de la Manche et pour qui une misérable auberge ou le palais de ducs farceurs sont les dérisoires théâtres de ses aventures grotesques. La dimension spatiale du Quichotte, malgré son caractère restreint et clairement topique, apparaît donc comme un élément-clé de la narration à tous les niveaux, diégétique, narratif, symbolique et idéologique.

\section{NOTES}

1. En réalité, les choses sont un peu plus complexes, comme le démontre longuement Gérard GENETTE dans son livre Palimpsestes, Paris, Le Seuil, coll. Poétique, 1982.

2. C'est la première phrase de l'introduction de l'Amadis de Gaule : « No muchos años después de la pasion de nostro redentor é salvador Jesucristo... », Biblioteca de Autores Españoles, Tomo XL, Libros de caballerías, Madrid, Atlas, 1963, p. 1.

3. Ernst Robert CURTIUS, Literatura europea y Edad Media Latina, Tomo 1, cap. X « El paisaje ideal », § 6 «El paraje ameno », Ed. Fondo de Cultura Económica, Madrid 1981, p. 280. (Titre original : Europaische literatur und lateinisches Mittelalter, A. Francke AG Verlag, de Berna, 1948. Trad. de Margit Frenk y Antonio ALATORRE). 
4. Comme le remarque le même CuRTius qui cite l'épisode du bois de Corpes du Cantar de mio Cid, un autre du Roland furieux de l'ARIOSTE et les livres de chevalerie en général dans lesquels le locus amoenus apparaît parfois au milieu de l'habituelle forêt sauvage. Ibid. p. 288-289 (texte et notes 38 et 39).

5. Terme utilisé par Jacques SOUBEYRoux dans son article «Le discours du roman sur l'espace. Approche méthodologique.", p. 16, in Lieux dits. Recherches sur l'espace dans les textes ibériques (XVIe-XXe siècles), Publications de l'Université de Saint-Étienne, 1993. SoubEYRouX reprend la notion et le terme du livre de Henri MitTeRRAND, Le discours du roman, Paris, P.U.F., 1980, p. 194. J'utiliserai dans la présente étude une partie de l'excellente méthodologie proposée par Jacques SOUBEYROUX dans l'article en question.

6. Quelques toponymes apparaissent çà et là dans le récit de la Diane de MONTEMAYOR : les Monts de Léon, la Lusitanie, la Galice, l'Andalousie (appelée "Vandalia») ou les noms de quelques fleuves comme l'Ezla, le Douro, etc.. Nous avons là un ancrage spatial très vague, bien que moins exotique que celui de la plupart des livres de chevalerie, mais la référence temporelle reste énigmatique. Il s'agit d'un récit intemporel situé dans un passé indéterminé localisé uniquement à travers les temps des verbes de la narration (imparfait et passé simple).

7. La plupart apparaissent dans l'histoire du capitaine captif, sans doute à cause du caractère nettement autobiographique de ce récit.

8. On ne saura donc jamais exactement où vivait don Quichotte. D'où les querelles séculaires entre divers villages de la Manche (Argamasilla de Alba ou Argamasilla de Calatrava par exemple qui revendiquent chacun l'honneur d'avoir été le berceau de l'ingénieux hidalgo.

9. Toposémie : Terme repris aussi de l'étude de Jacques SoubEYroux, op. cit., p. 17.

10. "Aquí pinta el autor todas las circunstancias de la casa de don Diego pintándonos en ellas lo que contiene una casa de un caballero labrador y rico ; pero al traductor desta historia le pareció pasar estas y otras semejantes menudencias en silencio, porque no venían bien con el propósito principal de la historia; la cual más tiene su fuerza en la verdad que en las frías digresiones. ", D.Q., P. II, chap. XVIII, p. 169. Dorénavant, je citerai le texte du Quichotte d'après l'édition de Luis Andrés MURILLo, Clásicos Castalia, Madrid, 1986.

11. "Llegaron, en estas pláticas, P. II, chap. 71, p. 572 al pie de una alta montaña... Corría por su falda un manso arroyuelo, $y$ hacíase por toda su redondez un prado tan verde y vicioso, que daba contento a los ojos que le miraban. Había por alli muchos árboles silvestres y algunas plantas y flores que hacían el lugar apacible. ", D. Q., P. I, chap. 25, p. 307.

12. "Entraron entre unos árboles amenos que poco desviados del camino estaban, donde, ..., se tendieron sobre la verde yerba... ", D. Q., P. II, chap. 71, p. 572.

13. "... vinieron a parar a un prado de fresca yerba, junto del cual corría un arroyo apacible y fresco; tanto, que convidó y forzó a pasar allí las horas de la siesta, que rigurosamente comenzaba a entrar. », D. Q., P. I, chap. 15, p. 190.

14. "... luego que vio la venta se le representó que era un castillo con sus cuatro torres y chapiteles de luciente plata, sin faltarle du puente levadiza y honda cava, con todos aquellos adherentes que semejantes castillos se pintan. », D. Q., P. I, chap. 2, p. 82.

15. «... si yo me pusiera ahora a decirlos (los trajes de las doncellas) como las historias nos los cuentan, sería nunca acabar... », D. Q., P. I, chap. 50, p. 585.

16. Selon la terminologie de Roland BARTHES qui oppose les informants, qui donnent de simples informations sans aucune autre fonction dans l'histoire que celle d'ancrer le récit dans l'espace et dans le temps, aux indices qui ont une fonction narrative parce qu'ils jouent un rôle indirect dans l'intrigue car ils renvoient implicitement à un caractère, un sentiment, une atmosphère, une idéologie. Cf Roland BARTHES, "Introduction à l'analyse structurale des récits", Communications nº 8 (1966), Paris, Le Seuil, « Points Essais », 1981. 
17. La différence entre "diégèse » et «narration» n'est pas toujours claire dans la critique contemporaine. Pour ma part, je vois la distinction entre « diégétique » et «narratif» comme celle qui existe entre les éléments qui composent l'univers de la fiction (coordonnées spatiotemporelles, personnages, atmosphères, etc..) et l'organisation du récit (disposition des séquences narratives, problèmes de niveaux narratifs, de mode, de voix, de focalisation, etc...).

18. Respectivement D. Q. I, chap. 8, 18, 19, 21.

19. D. Q., P. I, chap. 20, 23, 28, 50, et P. II, chap. 12.

20. « ... en el campo, como si fuera moro, ... al pie de la peña donde esta la fuente del alcornoque... » D. Q., P. I, chap. 12 .

21. Cf. Umberto ECO, Lector in fabula, Milano, Bompiani, 1979, trad. française, Ed. Grasset, 1985, particulièrement p. 95-106: «L'encyclopédie » et p. 110-116: « Le topic ».

22. Don Quichotte retourne chez lui dans une cage à la fin de la première partie et vaincu et désabusé à la fin de la seconde, Chrysostome revient dans son village pour y trouver le malheur et la mort tandis que Marcelle réussit à s'évader et à vivre librement la vie qu'elle a choisie et Léandre doit revenir chez elle pour être enfermée dans un couvent et pour oublier ses rêves d'évasion. Cf aussi la remarque de Roland BOURNEUF et Réal oUELLET dans leur ouvrage L'univers du roman, Éd. PUF 1972, p.129 : "Si l'errance est parfois une malédiction, pour CEdipe ou pour le peuple juif de la Bible, elle peut aussi signifier une libre réalisation de notre destin. » Pour plus de détails, voir les chapitres intitulés «L'espace » dans ma thèse de doctorat Fonction des nouvelles intercalées dans le roman espagnol au Siècle d'Or ", Cahiers de Narratologie $\mathrm{N}^{\circ}$ 4, Publications de la Faculté des Lettres et Sciences Humaines de Nice, 1991, p. 107-118 (pour le Guzmán de Alfarache de Mateo Alemán, avec les mêmes conclusions) et 267-276 (pour le Quichotte de 1605).

\section{AUTEUR}

\section{JEAN-LOUIS BRAU}

Université de Nice-Sophia Antipolis 